Supporting Information

\title{
Highly cross-linked, physiologically responsive, mechanically adaptive polymer networks made by photopolymerization
}

Baptiste Monney, Alicia G. Dibble, Dafni Moatsou, ${ }^{*}$ Christoph Weder

Adolphe Merkle Institute, University of Fribourg, Chemin des Verdiers 4, CH-1700

Fribourg, Switzerland

Corresponding Authors:

`E-mail: dafni.moatsou@kit.edu (D.M.), christoph.weder@unifr.ch (C.W.). 


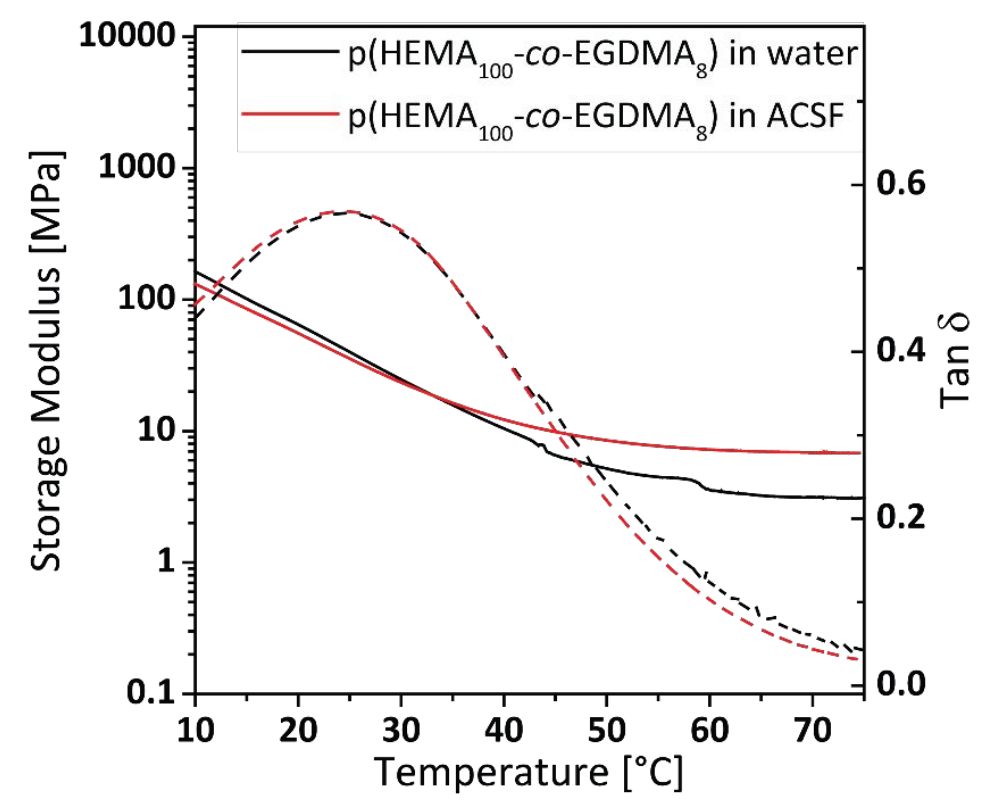

Figure S1: Storage modulus (solid curves) and loss tangent (dashed lines) of the waterswollen (black) and ACSF-swollen (red) p(HEMA ${ }_{100}-$ Co-EGDMA $\left.{ }_{8}\right)$. The samples used for this experiment were conditioned for 24 hours at $37^{\circ} \mathrm{C}$ in water or ACSF prior to measurement. 

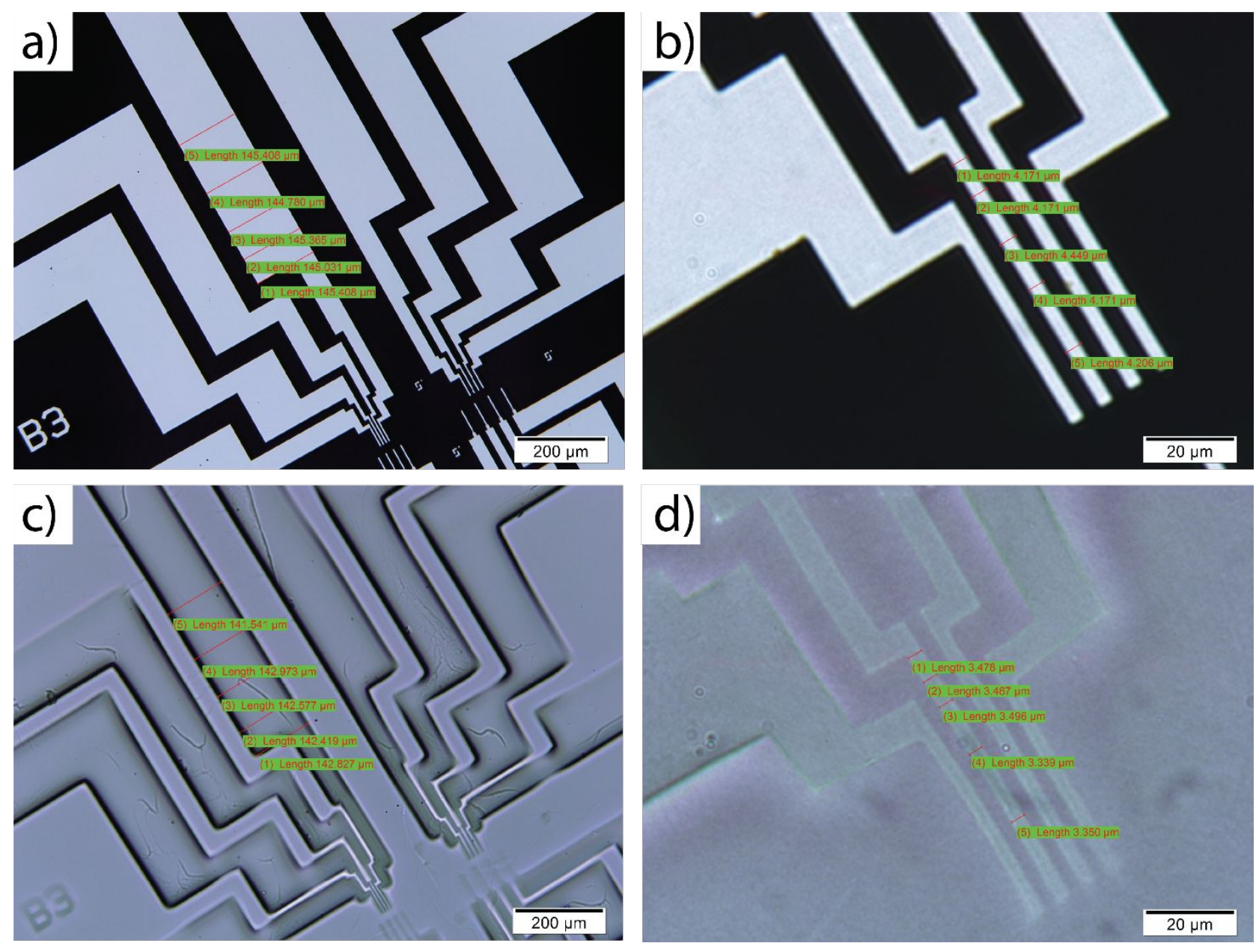

Figure S2: a) and b) optical microscope image of the photomask. c) and d) optical microscope image of the patterned mechanically adaptive polymer network on a glass slide. 


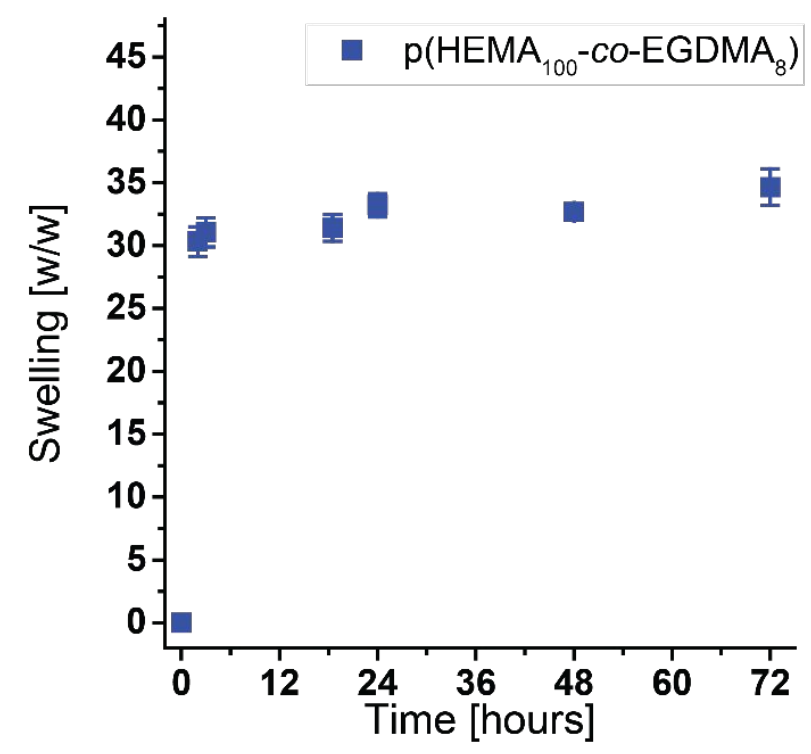

Figure S3: Swelling kinetics of poly $\left(\mathrm{HEMA}_{100^{-}} \mathrm{Co}-\mathrm{EGDMA}_{8}\right)$ in ACSF at $37^{\circ} \mathrm{C}$. 